\title{
Signatures of synchrony in pairwise count correlations
}

\section{Tatjana Tchumatchenko ${ }^{1,2,3}$, Theo Geisel ${ }^{1,2}$, Maxim Volgushev ${ }^{4,5,6}$ and Fred Wolf ${ }^{1,2}$}

\author{
1 Max Planck Institute for Dynamics and Self-Organization, Göttingen, Germany \\ 2 Bernstein Center for Computational Neuroscience Göttingen, Göttingen, Germany \\ ${ }^{3}$ Göttingen Graduate School for Neurosciences and Molecular Biosciences, Göttingen, Germany \\ 4 Institute of Higher Nervous Activity and Neurophysiology, Russian Academy of Sciences, Moscow, Russia \\ ${ }^{5}$ Department of Neurophysiology, Ruhr-University Bochum, Bochum, Germany \\ ${ }^{6}$ Department of Psychology, University of Connecticut, Storrs, CT, USA
}

\section{Edited by:}

Matthias Bethge, Max Planck Institute for Biological Cybernetics, Germany

\section{Reviewed by:}

Eric Shea-Brown, University of Washington, USA

Benjamin Lindner, Max Planck

Institute, Germany

*Correspondence:

Tatjana Tchumatchenko, Bernstein

Center for Computational

Neuroscience Göttingen, Bunsenstr.

10, 37073 Göttingen, Germany.

e-mail: tatjana@nld.ds.mpg.de
Concerted neural activity can reflect specific features of sensory stimuli or behavioral tasks. Correlation coefficients and count correlations are frequently used to measure correlations between neurons, design synthetic spike trains and build population models. But are correlation coefficients always a reliable measure of input correlations? Here, we consider a stochastic model for the generation of correlated spike sequences which replicate neuronal pairwise correlations in many important aspects. We investigate under which conditions the correlation coefficients reflect the degree of input synchrony and when they can be used to build population models. We find that correlation coefficients can be a poor indicator of input synchrony for some cases of input correlations. In particular, count correlations computed for large time bins can vanish despite the presence of input correlations. These findings suggest that network models or potential coding schemes of neural population activity need to incorporate temporal properties of correlated inputs and take into consideration the regimes of firing rates and correlation strengths to ensure that their building blocks are an unambiguous measures of synchrony.

Keywords: spike correlations, count correlations, population models, synchrony, correlation coefficient

\section{INTRODUCTION}

Coordinated activity of neural ensembles contributes a multitude of cognitive functions, e.g., attention (Steinmetz et al., 2000), encoding of sensory information (Stopfer et al., 1997; Galan et al., 2006), stimulus anticipation and discrimination (Zohary et al., 1994; Vaadia et al., 1995). Novel experimental techniques allow simultaneous recording of activity from a large number of neurons (Greenberg et al., 2008) and offer new possibilities to relate the activity of neuronal populations to sensory processing and behavior. Yet, understanding the function of neural assembles requires reliable tools for quantification, analysis and interpretation of multiple simultaneously recorded spike trains in terms of underlying connectivity and interactions between neurons.

As a first step beyond the analysis of single neurons in isolation, much attention has focused on the pairwise spike correlations (Schneidman et al., 2006; Macke et al., 2009; Roudi et al., 2009), their temporal structure and the influence of topology (Kass and Ventura, 2006; Kriener et al., 2009; Ostojic et al., 2009; Tchumatchenko et al., 2010). Pairwise neuronal correlations are traditionally quantified using count correlations, e.g., correlation coefficients (Perkel et al., 1967). However, it remains largely elusive how correlations present in the input to pairs of neurons are reflected in the count correlations of their spike trains. What are the signatures of input correlations in the count correlations? And vice versa, what conclusions about input correlations and interactions can be drawn on the basis of count correlations and their changes?

Here we address these questions using a framework of Gaussian random functions. We find that correlation coefficients can be a poor indicator of input synchrony for some cases of input correlations.
In particular, count correlations computed for time bins larger than the intrinsic temporal scale of correlations can vanish for some functional forms of input correlations. These potential ambiguities were not reported in previous studies of leaky integrate and fire models which focused on the analytically accessible choice of white noise input currents (de la Rocha et al., 2007; Shea-Brown et al., 2008).

The paper is organized as follows: we first introduce several common spike count measures (Section "Materials and Methods") and the statistical framework (Section "Results"). Then we study the zero time lag correlations (Section "Spike Correlations with Zero Time Lag") and the influence of the temporal structure of input correlations on measures of spike correlations (Section "Temporal Scale of Spike Correlations"). We show that spike count correlations can vanish despite the presence of input cross correlations (Section "Vanishing Count Covariance in the Presence of Cross Correlations"). Finally, we discuss potential consequences of our findings for the design of population models and the experimentally measured spike correlations.

\section{MATERIALS AND METHODS MEASURES OF CORRELATION}

The spike train $s_{i}(t)$ of a neuron $i$ is completely described by the sequence of spike times $t_{i}$. This description is often simplified using discrete bins of size $T$ (Figure 1). To describe pairwise spike correlations, several competing measures are used (Perkel et al., 1967; Svirskis and Hounsgaard, 2003; Schneidman et al., 2006; de la Rocha et al., 2007; Shea-Brown et al., 2008; Roudi et al., 2009). Here, we focus on the most commonly used measures of spike correlations: conditional 

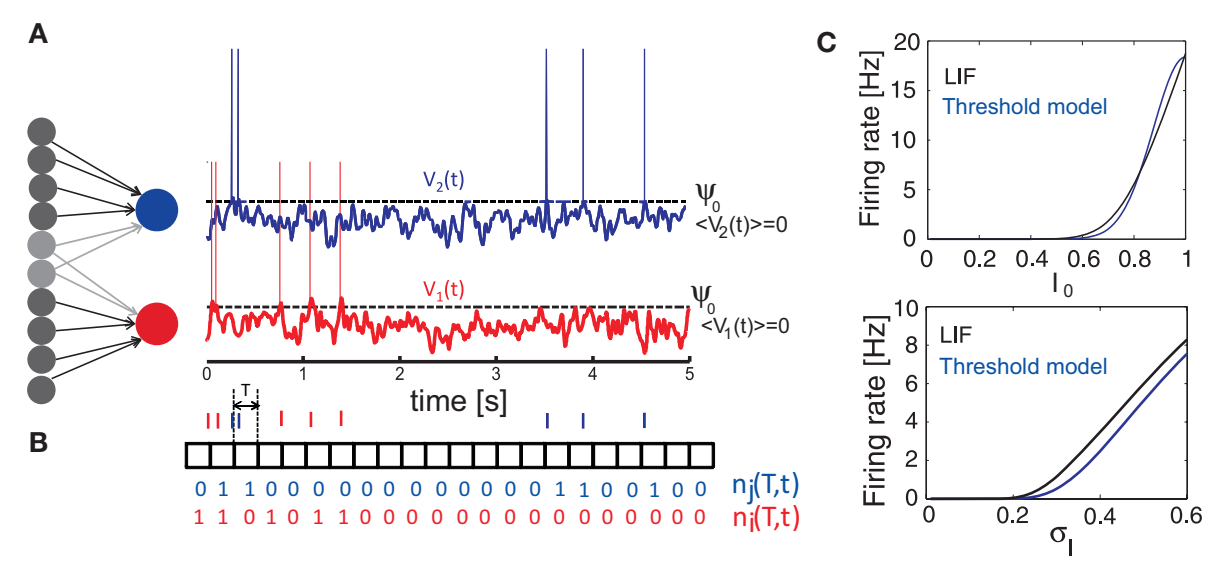

FIGURE 1 | Generation of spike trains and transformation to spike counts. (A) Generation of spike trains from correlated voltage traces of two neurons with common presynaptic partners. (B) Red and blue vertical bars indicate the spike trains of two neurons. Squares show the boundaries of bins with duration
T. $n_{i}(T, t)$ and $n_{j}(T, t)$ illustrate corresponding binned spike trains. (C) Firing rate vs. input current in the LIF model (first order solution) and the threshold model (Eq. 11) computed for $\sigma_{i}=0.25$ (top), $I_{0}=0.6$ (bottom) and $\psi_{0}=1, V_{r}=0$, $\tau_{M}=15 \mathrm{~ms}$ and $\tau_{f}=5 \mathrm{~ms}$. firing rate, correlation coefficient, normalized correlation coefficient and count covariance. We will consider the relation between these measures and their dependence on (1) the underlying input correlation strength, (2) firing rate, (3) temporal structure of spike trains, and (4) size of the time bin used to compute count correlations.

The spike timing correlations of two spike trains $s_{i}(t)$ and $s_{j}(t)$ are often quantified using the conditional firing rate function $v_{\text {cond }, i j}(\tau)$ (Binder and Powers, 2001; Tchumatchenko et al., 2010):

$\mathrm{v}_{\text {cond }, i j}(\tau)=\left\langle s_{i}(t) s_{j}(t+\tau)\right\rangle / \sqrt{v_{i} v_{j}}$

$\mathrm{v}_{\text {cond }}(\tau)=\mathrm{v}_{\text {cond }, i i}(\tau)=\left\langle s_{i}(t) s_{i}(t+\tau)\right\rangle / v_{i}$

Here $v_{i}$ and $v_{j}$ are the mean firing rates of neurons $i$ and $j$, respectively. Correlations within a spike train are described by the auto conditional firing rate $v_{\text {cond }}(\tau)$.

An alternative measure based on count correlations is the correlation coefficient $\rho_{i j}$ (Perkel et al., 1967; de la Rocha et al., 2007; Greenberg et al., 2008; Shea-Brown et al., 2008; Tetzlaff et al., 2008):

$\rho_{i j}=\frac{\operatorname{Cov}\left(n_{i}(T), n_{j}(T)\right)}{\sqrt{\operatorname{Var}\left(n_{i}(T), n_{i}(T)\right) \cdot \operatorname{Var}\left(n_{j}(T), n_{j}(T)\right)}}$

where $n_{i}(T)$ and $n_{j}(T)$ are spike counts of neuron $i$ and $j$ measured in synchronous time bins of width $T$, see Figure 1. A related measure of pairwise correlations is the normalized correlation coefficient $c_{i j}$ (Roudi et al., 2009). It determines pairwise interactions $J_{i j}$ in maximum entropy models of networks of $N$ neurons with average firing rate $\bar{v}$ (Schneidman et al., 2006; Roudi et al., 2009):

$$
\begin{aligned}
c_{i j} & =\frac{\operatorname{Cov}\left(n_{i}(T), n_{j}(T)\right)}{\left\langle n_{i}(T)\right\rangle\left\langle n_{j}(T)\right\rangle}=\frac{\operatorname{Cov}\left(n_{i}(T), n_{j}(T)\right)}{v_{i} v_{j} T^{2}} \\
J_{i j} & =\log \left(1+c_{i j}\right)+O(N \bar{v} T) .
\end{aligned}
$$

Covariance can be obtained via the integration of cross conditional firing rate $v_{\text {cond, } i j}(\tau)$ over the time bin $T$ :

$$
\begin{aligned}
\operatorname{Cov}\left(n_{i}(T), n_{j}(T)\right) & =\left\langle n_{i}(T), n_{j}(T)\right\rangle-\left\langle n_{i}(T)\right\rangle\left\langle n_{j}(T)\right\rangle \\
& =\left\langle\int_{0}^{T} s_{i}\left(x_{1}\right) d x_{1} \int_{0}^{T} s_{j}\left(x_{2}\right) d x_{2}\right\rangle-v_{i} v_{j} T^{2} \\
& =\int_{-T}^{T} \sqrt{v_{i} v_{j}}\left(v_{\text {cond }, i j}(t)-\sqrt{v_{i} v_{j}}\right)(T-|t|) d t .
\end{aligned}
$$

The count variance can be obtained from the auto conditional firing rate $v_{\text {cond }}(\tau)$ :

$\operatorname{Var}\left(n_{i}(T), n_{i}(T)\right)=v_{i} \cdot T+2 \cdot \int_{0}^{T} v_{i}\left(v_{\text {cond }}(t)-v_{i}\right)(T-|t|) d t$.

For bin sizes smaller than the intrinsic time constant $(T<\tau$, see Eq. 14), we can directly relate conditional firing rate $v_{\text {cond, }, j}(\tau)$ and the correlation coefficient $\rho_{i j}$

$\rho_{i j, T<\tau_{s}} \approx \frac{\sqrt{v_{i} v_{j}} \cdot\left(v_{\text {cond }, i j}(0)-\sqrt{v_{i} v_{j}}\right) T^{2}}{\sqrt{v_{i} v_{j}} T \sqrt{\left(1-v_{i} \cdot T\right)\left(1-v_{i} \cdot T\right)}}=\left(v_{\text {cond }, i j}(0)-\sqrt{v_{i} v_{j}}\right) T$

$c_{i j, T<\tau_{s}} \approx \frac{\sqrt{v_{i} v_{j}} \cdot\left(v_{\text {cond }, i j}(0)-\sqrt{v_{i} v_{j}}\right) T^{2}}{v_{i} v_{j} T^{2}}=\frac{v_{\text {cond }, i j}(0)-\sqrt{v_{i} v_{j}}}{\sqrt{v_{i} v_{j}}}$.

In this limit, the properties of $\rho_{i j}, c_{i j}$ are largely determined by $v_{\text {cond }, i j}(0)$. Several experimental studies used bin sizes ranging from $T=0.1$ to $1 \mathrm{~ms}$, which are compatible with this $T$-regime of correlation coefficients (e.g., Lampl et al., 1999; Takahashi and Sakurai, 2006). 
The quantities presented here all measure different aspects of spike correlations and can potentially have different computational properties. Furthermore, each of the quantities can exhibit a nonlinear dependence on firing rate, input statistics or bin size. Below, we consider these measures of spike correlations, as well as their dependence on firing rate, input statistics and bin size.

\section{RESULTS}

To access spike correlations in a pair of neurons, we use the framework of correlated, stationary Gaussian processes to model the voltage potential $V(t)$ of each neuron. This approach generates voltage traces with statistical properties consistent with cortical neurons (Azouz and Gray, 1999; Destexhe et al., 2003). The simplest conceivable model of spike generation from a fluctuating voltage $V(t)$ identifies the spike times $t_{j}$ with upward crossings of a threshold voltage (Rice, 1954; Jung, 1995; Burak et al., 2009). The times $t_{j}$ determine the spike train:

$s(t)=\Sigma_{j} \delta\left(t-t_{j}\right)=\delta\left(V(t)-\psi_{0}\right)|\dot{V}(t)| \theta(\dot{V}(t))$,

where $\psi_{0}$ is the threshold voltage, and $\delta(\cdot)$ and $\theta(\cdot)$ are the Dirac delta and Heaviside theta functions, respectively. Each neuron has a stationary firing rate $v=\langle s(t)\rangle$. We model $V(t)$ by a random realization of a stationary continuous correlated Gaussian process $V(t)$ (Azouz and Gray, 1999; Destexhe et al., 2003) with zero mean and a temporal correlation function $C(\tau)$, which decays for larger time lags $\tau$.

$C(\tau)=\langle V(t) V(t+\tau)\rangle=\langle V(0) V(\tau)\rangle,\langle V(t)\rangle=0$

$\langle\cdot\rangle$ denotes the ensemble average. We assume a smooth $C(\tau)$ such that $C^{n}(0)$ exist for $n \leq 6$ and the rate of threshold crossings is finite (Stratonovich, 1964). All other properties of $C(\tau)$ can be freely chosen. This makes our formal description applicable to a large class of models, each of which is characterized by a particular choice $C(\tau)$. For simulations using digitally synthesized Gaussian processes (Prichard and Theiler, 1994) and numerical integration of Gaussian integrals (e.g., Wolfram Research, 2009) we used a correlation function compatible with power spectra of cortical neurons (Destexhe et al., 2003):

$C(\tau)=\sigma_{V}^{2} \cosh \left(\tau / \tau_{s}\right)^{-1}$

In cortical neurons in vivo the temporal width of $C(\tau)$ can from 10 to $100 \mathrm{~ms}$ (Azouz and Gray, 1999; Lampl et al., 1999). We characterize the temporal width of $C(\tau)$ using the correlation time constant $\tau_{s}$ :

$\tau_{s}=\sqrt{C(0) /\left|C^{\prime \prime}(0)\right|}$

Note, that the correlation time $\tau_{s}$ as defined in Eq. 14 is close to a commonly used definition of autocorrelation time $\tau_{a}=\int_{0}^{\infty} C(\tau) / \sigma_{V}^{2}$. For $C(\tau)$ as in Eq. $13 \tau_{\mathrm{a}}=\pi \tau_{\mathrm{s}} / 2$. The correlation time $\tau_{s}$ and the threshold $\psi_{0}$ determine the firing rate $v$ :

$v=\frac{\exp \left[-\psi_{0}^{2} /\left(2 \sigma_{V}^{2}\right)\right]}{2 \pi \tau_{s}}$.

The firing rate $v$ is the rate of positive threshold crossings, which is equivalent to half of the Rice rate of a Gaussian process (Rice, 1954). For non-Gaussian processes the rate of threshold crossings can deviate from Eq. 15 and there is no general approach for obtaining $v$ in this case (Leadbetter et al., 1983). We note, that the firing rate $v$ of a neuron depends only on two parameters: the correlation time and the threshold-to-variance ratio, but not on the specific functional choice of the correlation function. Hence, processes with the same correlation time but with a different functional form of $C(\tau)$ will have the same mean rate of spikes, though their spike auto and cross correlations can differ significantly. Our framework can be expected to capture neural activity in the regime where the mean time between the subsequent spikes is much longer than the decay time of the spike triggered currents. This occurs if the spikes are sufficiently far apart and the spike decision is primarily determined by the stationary voltage statistics rather than spike evoked currents. Therefore, this model should only be used in the fluctuation driven, low firing rate $v<1 /(2 \pi \tau)$ regime, which is important for cortical neurons (Greenberg et al., 2008).

The leaky integrate and fire (LIF) model (Brunel and Sergi, 1998; Fourcaud and Brunel, 2002) has a similar spike generation mechanism. To compare both models, we study the transformation of input current to spikes. The LIF neuron driven by OrnsteinUhlenbeck current $I(t)$ with time constant $\tau_{I}$ can be described by

$\tau_{M} \dot{V}(\tau)=-V+I_{0}+I(t)$

where $\tau_{M}$ is the membrane time constant and $I_{0}$ is the mean input current. When $V(t)$ reaches the threshold $\psi_{0}$, the neuron emits a spike, and $V(t)$ is reset to $V_{r}$. The LIF model mainly differs from our framework by the presence of reset after each spike. For low firing rates, where the reset has little influence on the following spike, the threshold model and the LIF model can be expected to yield equivalent results. In Figure 1C we compare the first order firing rate approximation (first order in $\sqrt{\tau_{I} / \tau_{M}}$ ) of a LIF neuron driven by colored noise, which can be obtained via involved Fokker-Planck calculations (Brunel and Sergi, 1998; Fourcaud and Brunel, 2002) and the firing rate of the corresponding threshold neuron $v=\left(2 \pi \sqrt{\tau_{I} \tau_{M}}\right)^{-1} \exp \left(-\left(I_{0}-\psi_{0}\right)^{2}\left(\tau_{I}+\tau_{M}\right) /\left(2 \sigma_{I}^{2} \tau_{I}\right)\right)$. In general, the details of the spike generating model can have a strong effect on current susceptibility and spike correlations (Vilela and Lindner, 2009). However, we find that both models have a very similar current susceptibility for a range of input currents and spike correlations derived in the forthcoming sections are consistent with the corresponding correlations in the LIF model, e.g., firing rate dependence of weak cross correlations (de la Rocha et al., 2007; Shea-Brown et al., 2008), the influence of noise mean and variance on the firing rates and spike correlations (Brunel and Sergi, 1998; de la Rocha et al., 2007; Ostojic et al., 2009), sublinear dependence of correlation coefficients on input strength (Moreno-Bote and Parga, 2006; de la Rocha et al., 2007).

We include cross correlation between two spike trains $i$ and $j$ via a common component in $V_{i}(t)$ and $V_{j}(t), r>0$ :

$V_{i}(t)=\sqrt{1-r} \xi_{i}(t)+\sqrt{r} \xi_{c}(t)$
$V_{j}(t)=\sqrt{1-r} \xi_{j}(t)+\sqrt{r} \xi_{c}(t)$.

where $\xi_{c}$ denotes the common component and $\xi_{i}$, $\xi_{j}$ are the individual noise components. In a Gaussian ensemble any expectation value is determined by pairwise covariances only. Thus 
all pairwise correlations are determined by the joint Gaussian probability density $p(\vec{k})=\exp \left(-\vec{k}^{T} C^{-1} \vec{k} / 2\right) /\left(4 \pi^{2} \sqrt{\text { Det } C}\right)$ of $\vec{k}=\left(V_{i}(0), \dot{V}_{i}(0), V_{j}(\tau), \dot{V}_{j}(\tau)\right)$, where

$$
C=\left(\begin{array}{cccc}
\sigma_{V_{i}}^{2} & 0 & C_{i j}(\tau) & C_{i j}^{\prime}(\tau) \\
0 & \sigma_{\dot{V}_{i}}^{2} & -C_{i j}^{\prime}(\tau) & -C_{i j}^{\prime \prime}(\tau) \\
C_{i j}(\tau) & -C_{i j}^{\prime}(\tau) & \sigma_{V_{i}}^{2} & 0 \\
C_{i j}^{\prime}(\tau) & -C_{i j}^{\prime \prime}(\tau) & 0 & \sigma_{\dot{V}_{j}}^{2}
\end{array}\right) .
$$

Matrix entries are covariances $C_{x y}=\left\langle k_{x} k_{y}\right\rangle$ with $C_{i j}=r C(\tau)$. Below, we calculate the conditional firing rate $v_{\text {cond, } i j}(\tau)$ (Eqs 1 and 11) for several important limits.

\section{SPIKE CORRELATIONS WITH ZERO TIME LAG}

The above framework allows one to derive an analytical expression for the cross conditional firing rate with zero time lag, $v_{\text {cond }, i j}(0)$. Via Eqs 5, 9 and $10 v_{\text {cond,ij }}(0)$ can be related to $c_{i j}, \rho_{i j}$ and $J_{i j}$. For a pair of statistically identical neurons with $\left(v=v_{1}=v_{2}\right) \cdot v_{\text {cond }, i j}(0)$ in Eq. 1 can be solved by transforming the correlation matrix $C$ (Eq. 18) into a block diagonal form via a variable transformation:

$$
\begin{aligned}
& \Sigma=\frac{V_{1}(0)+V_{2}(\tau)}{\sqrt{2} \sqrt{\sigma_{V}^{2}+r C(\tau)}}, \dot{\Sigma}=\frac{\dot{V}_{1}(0)+\dot{V}_{2}(\tau)}{\sqrt{2} \sqrt{\sigma_{\dot{V}}^{2}-r C^{\prime \prime}(\tau)}}, \\
& \Delta=\frac{V_{1}(0)-V_{2}(\tau)}{\sqrt{2} \sqrt{\sigma_{V}^{2}-r C(\tau)}}, \dot{\Delta}=\frac{\dot{V}_{1}(0)-\dot{V}_{2}(\tau)}{\sqrt{2} \sqrt{\sigma_{\dot{V}}^{2}+r C^{\prime \prime}(\tau)}} .
\end{aligned}
$$

The matrix $C$ is then the identity matrix for $\tau=0$, and $\sum=\sqrt{2} \psi_{0} / \sqrt{\sigma_{V}^{2}+r \sigma_{V}^{2}}, \Delta=0$. We obtain:

$\mathrm{v}_{\text {cond }, i j}(0)=\int_{-\infty}^{\infty} \int_{-\infty}^{\infty} d \dot{\Sigma}, d \dot{\Delta} \exp \left(-\left(\frac{\psi_{0}^{2}}{\sigma_{V}^{2}(1+r)}+\frac{\dot{\Delta}^{2}+\dot{\Sigma}^{2}}{2}\right)\right)$

$\times \frac{\sigma_{\dot{V}}^{4} \sqrt{\left(1-r^{2}\right)}}{v 8 \pi^{2} \sqrt{\operatorname{Det} C}}\left(\dot{\Sigma}^{2}(1+r)-\dot{\Delta}^{2}(1-r)\right)$

$\times \theta\left(\frac{\sigma_{\dot{V}}}{\sqrt{2}}(\dot{\Sigma} \sqrt{(1+r)}+\dot{\Delta} \sqrt{(1-r)})\right) \times \theta\left(\frac{\sigma_{\dot{V}}}{\sqrt{2}}(\dot{\Sigma} \sqrt{(1+r)}-\dot{\Delta} \sqrt{(1-r)})\right)$

$=\frac{1}{4 \pi^{2} v \tau_{s}^{2}} \exp \left(\frac{-\psi_{0}^{2}}{\sigma_{V}^{2}(1+r)}\right)\left[1+\frac{2 r \cdot \arctan \left(\sqrt{\frac{1+r}{1-r}}\right)}{\sqrt{1-r^{2}}}\right]$.
Equation 19 (Figure 3A) shows, as expected, that $v_{\text {cond }, i j}(0)$ increases with increasing strength of input correlations $r$. Since both correlation coefficients $\rho_{i j}$, and normalized correlation coefficient $c_{i j}$ are proportional to $v_{\text {cond }, i j}(0)$ (Eqs 9 and 10), both measures also increase with increasing $r$, which is consistent with experimental findings (Binder and Powers, 2001; de la Rocha et al., 2007). However, the functional form of $r$-dependence and the sensitivity to the firing rate $v$ of $c_{i j}$ and $\rho_{i j}$ are different (Figure 2). The normalized correlation coefficient $c_{i j}$ and pairwise coupling $J_{i j}$ are both inversely proportional to $v$, and thus decrease with increasing $v$ for any value of $r$ (Eqs 4 and 5; Figure 2B). Notably, we find that $c_{i j}$ can be normalized to $c_{i j} \rightarrow c_{i j} \cdot(v T)$ to yield a less ambiguous measure of the input correlation strength (Eqs 4 and 10; Figures 3C,D). Additionally, we find that the firing rate dependence of $\rho_{i j}$ is different for the weak and strong correlations.

Equation 19 further exposes one important feature of $v_{\text {cond }, i j}(0)$, and thus of $c_{i j}$ and $\rho_{i j}$ for small time bins: all three measures depend on the temporal scale of the input correlations $\left(\tau_{s}\right)$, but not on the functional form of input correlation $C(\tau)$. Thus, changes in $v_{\text {cond }, i j}(0)$ and correlation coefficient $\rho_{i j}$ can be interpreted as a change of the strength of underlying input correlation strength, if a firing rate modification can be excluded.

In the linear $r$-regime, the analytical expression for $v_{\text {cond, }, j}(0)$ can be further simplified:

$\mathrm{v}_{\text {cond }, i j}(0) \approx \mathrm{v}\left(1+\frac{r}{2}\left(\pi+4\left|\log \left(\mathrm{v} 2 \pi \tau_{s}\right)\right|\right)\right)$.

In this limit, $v_{\text {cond }, i j}(0)$ shows a strong dependence on the firing rate $\boldsymbol{v}$ (Figure $\mathbf{3 A}$, right, Figure $\mathbf{2 A}$, top). This dependence is remarkably similar to the firing rate dependence found previously in vitro and in vivo in cortical neurons and LIF models (de la Rocha et al., 2007; Greenberg et al., 2008; Shea-Brown et al., 2008).

In the limit of strong input correlations, Eq. 19 can be simplified to:

$\mathrm{v}_{\text {cond }, i j}(0) \approx \frac{1}{2 \sqrt{2} \sqrt{1-r} \tau_{s}}$.

In this regime, $v_{\text {cond, } i j}(0)$ does not depend on the firing rate $v$ (Amari, 2009). Furthermore, for strong input correlations and small bin sizes $T$ the correlation coefficient $\rho_{i j}$ also changes only

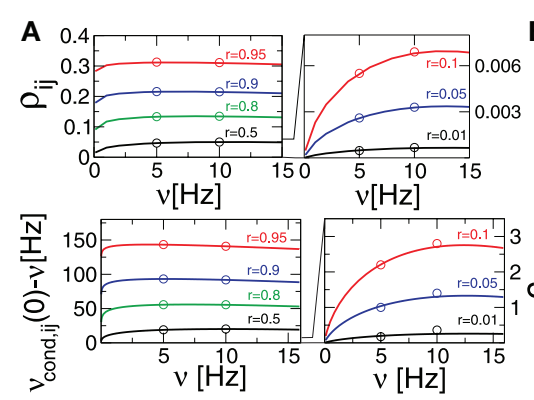

FIGURE 2 | Dependence of correlation coefficient $\rho_{i j}$ and conditional rate $\mathbf{v}_{\text {cond di }}(\mathbf{0})$ on firing rate and correlation strength. (A, top) $\rho_{i j} v s . v,(\mathbf{A}$, bottom) $v_{\text {cond,if }}\left(\right.$ (O) vs. $v$, as in Eq. 19. (B, top) Pairwise couplings $J_{i j}$ vs. $v$, as in Eq. 5. (B,

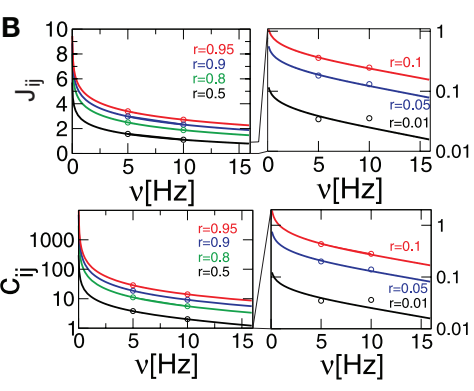

bottom) $c_{i j}$ vs. v. All quantities are computed for $\tau_{s}=10 \mathrm{~ms}, C(\tau)$ as in Eq. 13 and $v=v_{1}=v_{2}$; circles denote the corresponding simulation results. $\rho_{i j}, c_{i j}$ and $J_{i j}$ are computed for $T=\tau_{\mathrm{s}} / 4$. 


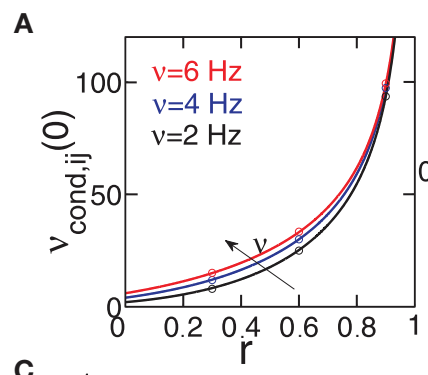

C
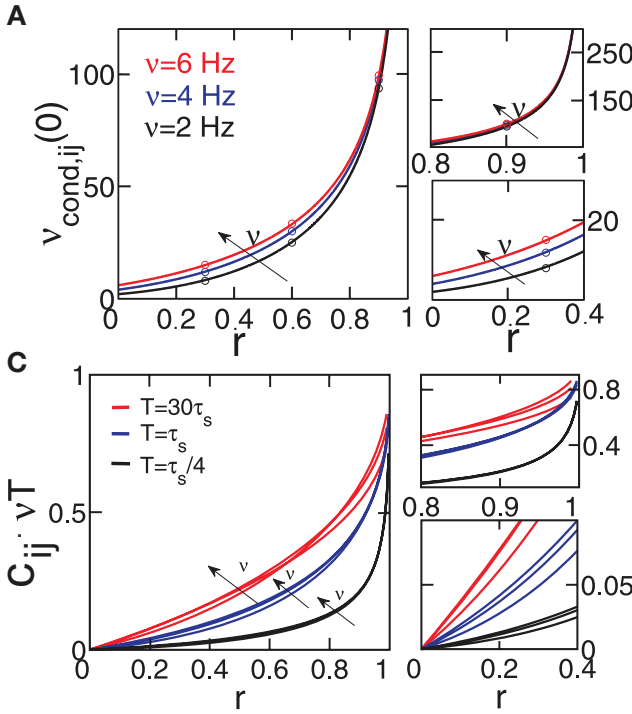

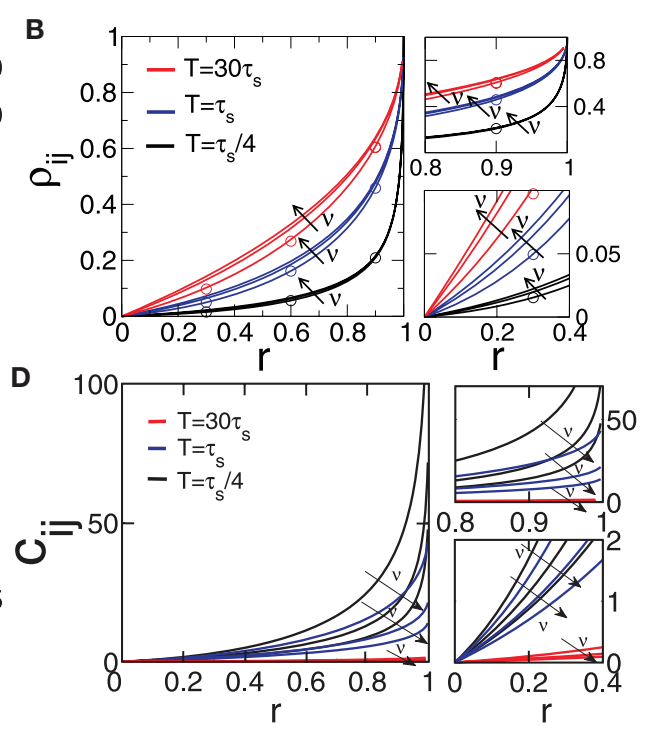

computed for $C(\tau)$ as in Eq. 13, correlation time $\tau_{s}=10 \mathrm{~ms}$ and three firing rates $v=2,4,6 \mathrm{~Hz}, v=v_{1}=v_{2}$; circles denote simulation results for the corresponding parameters.
FIGURE 3 | Dependence of spike correlation measures on firing rate $v$ and correlation strength $\boldsymbol{r}$. (A) $v_{\text {cond }, i j}(0)$ vs. $r$ (B) $\rho_{i j}$ vs. $r$ for bin widths $T=30 \tau_{s}$ (red), $T=\tau_{s}$ (blue), $T=\tau_{s} / 4$ (black). (C) $c_{i j} v T$ vs. $r$. (D) $c_{i j}$ vs. r. All quantities are marginally over a range of firing rates $(0<v<15 \mathrm{~Hz}$, Figure 2A), since it depends linearly on $v_{\text {cond }, i j}(0)$. Note, as $r$ is approaching 1 the temporal width of $v_{\text {cond, }, i j}(\tau)$ is approaching 0 and the peak $v_{\text {cond,ij }}(0)$ diverges, corresponding to the delta peak in the autoconditional firing rate $v_{\text {cond }}(\tau)$ which results from the self-reference of a spike. For $r \approx 1$, almost every spike in one train has a corresponding spike in the other spike train, however these two are jittered. The temporal jitter of the spikes can be characterized by the peak of the conditional firing rate $\nu_{\text {cond, } 12}(\tau)=1 /\left(2 \sqrt{2} \sqrt{1-r} \tau_{s}\right)-3 \tau^{2} /\left(8 \sqrt{2}(1-r)^{3 / 2} \tau_{s}^{3}\right)+$ $O\left[\left(\tau /\left(\sqrt{1-r} \tau_{s}\right)\right)^{4}\right]$ and its temporal width $\propto \sqrt{2} \sqrt{1-r} \tau_{s}$, both of which are threshold and firing rate independent in this limit. Notably, the threshold independence and the dependence on temporal scale of input correlations are consistent with previous experimental findings on spike reliability (Mainen and Sejnowski, 1995).

\section{TEMPORAL SCALE OF SPIKE CORRELATIONS}

So far we considered only spike correlations occurring with zero time lag. However, spike correlations can also span across significant time intervals (Azouz and Gray, 1999; Destexhe et al., 2003). The temporal structure of spike correlations, as reflected in the conditional firing rate $v_{\text {cond }, i j}(\tau)$, can induce temporal correlations within and across time bins and could potentially alter count correlations. To capture correlations with a non-zero time lag, spike correlation measures are calculated for time bins $T$ spanning tens to hundreds of milliseconds, e.g., $20 \mathrm{~ms}$ (Schneidman et al., 2006), 30-70 ms (Vaadia et al., 1995), $192 \mathrm{~ms}$ (Greenberg et al., 2008) and 2 s (Zohary et al., 1994). For time bins longer than the time constant of the input correlations, measures of correlations become sensitive to the temporal structure of $v_{\text {cond }, i j}(\tau)$. Moreover, the values of $\rho_{i j}$ and $c_{i j}$ depend on the bin size $T$ used for their calculation. Figure 3 shows how dependence of $\rho_{i j}$ and $c_{i j}$ on the firing rate is altered by a change in bin size. Increasing the bin size leads to the increase of the calculated correlation coefficient $\rho_{i j}$, and also increases the sensitivity of $\rho_{i j}$ to the firing rate. The fact that increasing $T$ brings the calculated correlation coefficient closer to the underlying input correlation $r$ could justify the use of long time bins in the above studies. But do correlation coefficients always increase with increasing time bins? To further clarify how the temporal structure of input correlations influences the temporal correlations within and across spike trains, we investigate the covariance of spike counts recorded at different times

$$
\begin{aligned}
& \operatorname{Cov}\left(n_{i}(T, t), n_{j}(T, t+\tau)\right)=\left\langle n_{i}(T, 0) n_{j}(T, \tau)\right\rangle-v_{i} v_{j} T^{2} \\
& =\int_{-T}^{T} \sqrt{v_{i} v_{j}}\left(v_{\text {cond }, i j}(\tau+t)-\sqrt{v_{i} v_{j}}\right)(T-|t|) d t
\end{aligned}
$$

where $n_{i}(T, t)$ and $n_{i}(T, t+\tau)$ are the spike counts of neurons $i, j$ measured in time bins of the same duration $T$, but shifted by the time lag $\tau$. For each time lag $\tau$, covariance of the spike counts can be calculated using $v_{\text {cond }, i j}(\tau)$ (Eq. 1). Below, we will first address the temporal structure of auto correlations in a spike train, and then consider the cross correlations between spike trains.

\section{The auto conditional firing rate $v_{\text {cond }}(\tau)$}

For large time lags $\tau$ we expect the auto conditional firing rate to approach the stationary rate but to deviate from it significantly for small time lags. Of particular importance for population models is the limit of small but finite $\tau$, which determines the time scale on which adjacent time bins are correlated. At $\tau=0$, the auto conditional firing rate has a $\delta$-peak reflecting the trivial auto correlation of each spike with itself. In the limit of small but finite time lag $\left(0<\tau<\tau_{s}\right)$ we find a period of intrinsic silence, where the leading order $\propto \tau^{4}$ is independent of a particular functional 
choice of $C(\tau)$. We solve $v_{\text {cond }}(\tau)$ (Eq. 2) by transforming the correlation matrix in Eq. 18 into a block diagonal form using new variables

$$
\begin{aligned}
& \sum=\frac{V(0)+V(\tau)}{\sqrt{2} \sqrt{\sigma_{V}^{2}+C(\tau)}}, \dot{\Sigma}=\frac{\dot{V}(0)+\dot{V}(\tau)}{\sqrt{2} \sqrt{\sigma_{\dot{V}}^{2}-C^{\prime \prime}(\tau)}}, \\
& \Delta=\frac{V(0)-V(\tau)}{\sqrt{2} \sqrt{\sigma_{V}^{2}-C(\tau)}}, \dot{\Delta}=\frac{\dot{V}(0)-\dot{V}(\tau)}{\sqrt{2} \sqrt{\sigma_{\dot{V}}^{2}+C^{\prime \prime}(\tau)}} .
\end{aligned}
$$

Then only few elements of the corresponding symmetric density matrix $C_{\Sigma, \dot{\Delta}, \dot{\Sigma}, \Delta}$ remain non-zero: the diagonal elements $C_{\Sigma, \dot{\Delta}, \dot{\Sigma}, \Delta_{i i}}=1$, $i \in\{1,2,3,4\}$ and the non-diagonal elements

$$
\begin{aligned}
& C_{\Sigma, \dot{\Delta}, \dot{\Sigma}, \Delta_{12}}=\frac{-C^{\prime}(\tau)}{\sqrt{\sigma_{V}^{2}+C(\tau)} \sqrt{\sigma_{\dot{V}}^{2}+C^{\prime \prime}(\tau)}}, \\
& C_{\Sigma, \dot{\Delta}, \dot{\Sigma}, \Delta_{34}}=\frac{C^{\prime}(\tau)}{\sqrt{\sigma_{V}^{2}-C(\tau)} \sqrt{\sigma_{\dot{V}}^{2}-C^{\prime \prime}(\tau)}} .
\end{aligned}
$$

For $C(\tau)$ as in Eq. 13 we obtain a simple analytical expression in the limit of $0<\tau<\tau$ :

$v_{\text {cond }}(\tau)=\frac{v^{1 / 4}}{3 \cdot\left(2 \pi \tau_{s}\right)^{\frac{3}{4}}} \cdot\left(\tau / \tau_{s}\right)^{4}$.

This equation shows that $v_{\text {cond }}(\tau)$ depends on the temporal structure of a neuron's input and firing rate, Figure 4B. Respectively, the silence period after each spike depends on the functional form and time constant of the voltage correlation function $C(\tau)$ and firing rate (Figures $4 B$ and 5A). Figure 4B illustrates $v_{\text {cond }}(\tau)$ obtained using numerical integration of Gaussian probability densities (e.g., Wolfram Research, 2009), $v_{\text {cond }}(\tau)$ obtained from simulations of digitally synthesized Gaussian processes (Prichard and Theiler,
1994) and the $\tau<\tau_{s}$ approximation in Eq. 23. In this framework, the silence period after each spike mimics the refractoriness present in real neurons (Dayan and Abbott, 2001).

\section{Count correlations within a spike train}

Here we study how the input correlations shape the temporal structure of spike autocorrelations. In particular, we focus on how the input correlations and spike autocorrelations are reflected in count correlations within a spike train. The silence period after a spike is reflected in vanishing $v_{\text {cond }}(\tau)$ for $0<\tau<\tau_{s}$ and results in negative covariation of spike counts in adjacent time bins. We find that the relation between $v_{\text {cond }}(\tau)$ and spike count covariance is most salient for higher firing rates (Figure 4C, $10 \mathrm{~Hz}$ ). For small time bins, the covariance mimics the functional form of $v_{\text {cond }}(\tau)$ for time bins covering several time constants. Plots of spike count covariance calculated for increasing bin sizes $T$ reveal an important feature of count correlations: covariance of adjacent bins persists even when the bin size is increased well over the time scale of intrinsic correlations $\left(T>>\tau_{s}\right)$, Figure 4 . This suggests that avoiding statistical dependencies associated with neuronal refractoriness by choosing longer time bins (Shlens et al., 2006) might not be possible, particularly for higher firing rate neurons. We conclude that temporal count correlations within a spike train generally need to be considered in the design of population models.

\section{Cross conditional firing rate $\mathrm{v}_{\text {cond }, i j}(\tau)$}

We explore the temporal structure of spike correlations in a weakly correlated pair of statistically identical neurons $\left(v=v_{1}=v_{2}\right)$. This is an important regime for cortical neurons in vivo (Greenberg et al., 2008; Smith and Kohn, 2008). To solve $v_{\text {cond }, i j}(\tau)$ (Eq. 1), we expand the probability density $p\left(V_{1}(t), \dot{V}_{1}(t), V_{2}(t+\tau), \dot{V}_{2}(t+\tau)\right)$ using a von Neumann series of the correlation matrix $C$ in Eq. 18. We obtain $v_{\text {cond }, i j}(\tau)$ up in linear order
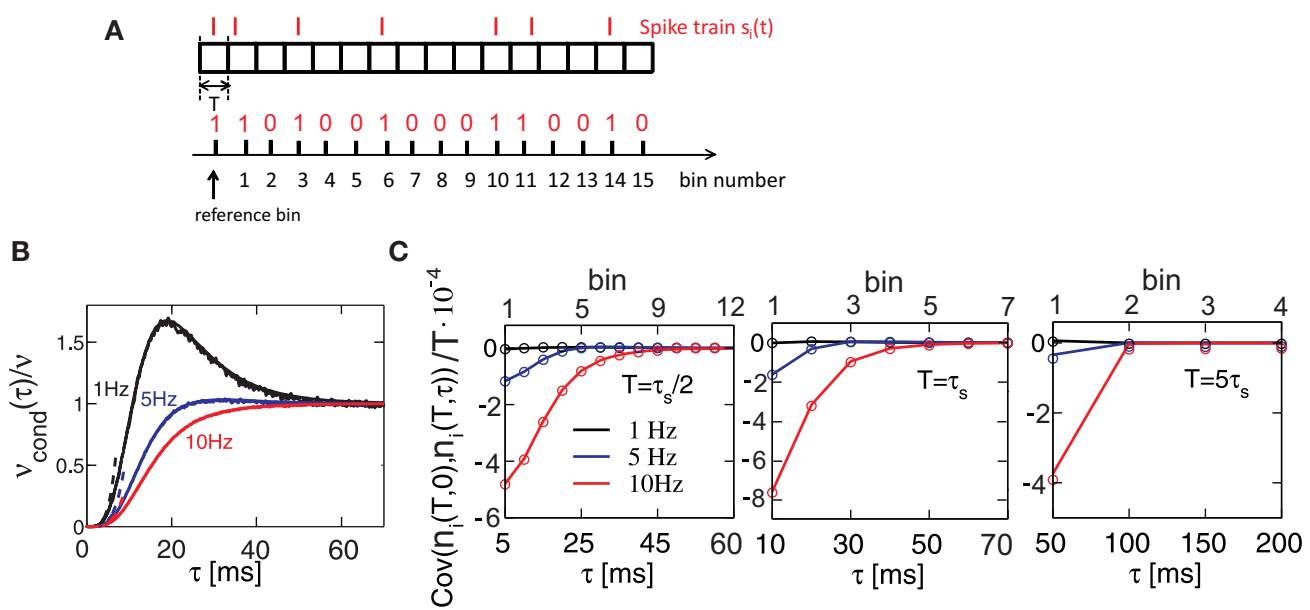

FIGURE 4 | Spike correlations and count correlations within a spike train. (A) Example of a binned spike train $s_{i}(t)$, bins numbered with respect to a reference time bin. (B) $v_{\text {cond }}(\tau)$ vs. $\tau$ for $\tau=10 \mathrm{~ms}$, numerical solution and simulations for the firing rates $v=1 \mathrm{~Hz}$ (black), $5 \mathrm{~Hz}$ (blue) and $v=10 \mathrm{~Hz}$ (red) are superimposed. Dotted lines denote the corresponding solutions for small $\tau$ (Eq. 23). (C) $\operatorname{Cov}\left(n_{i}(T, 0), n(T, \tau)\right) / T$ vs. $\tau$ for $\tau_{s}=10 \mathrm{~ms}$, time bin $T=\tau_{s} / 2=5 \mathrm{~ms}$ (left), $T=10 \mathrm{~ms}=\tau_{s}$ (middle), $T=5 \tau_{\mathrm{s}}=50 \mathrm{~ms}$ (right). Circles denote the corresponding simulation points, adjacent time bins are denoted by the first points on the time axis. All spike correlations are computed for $C(\tau)$ as in Eq. 13. 


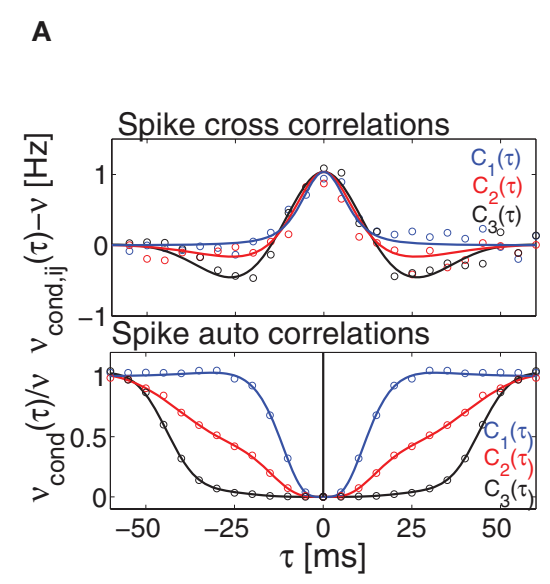

FIGURE 5 | Influence of temporal structure on pairwise spike correlations. (A) Spike cross correlations $v_{\text {cond }, i j}(\tau)$ and auto correlations $v_{\text {cond }}(\tau)$ for three voltage correlation functions $C_{j}(\tau)$. (B) Voltage correlations $C_{1}(\tau)=\sigma_{V}^{2} \cosh \left(\tau / \tau_{s}\right)^{-1}$ (blue), $C_{2}(\tau)=\sigma_{V}^{2} \cosh \left(\tau /\left(\sqrt{2 \tau_{s}}\right)\right)^{-1} \cos \left(\tau / \sqrt{2 \tau_{s}}\right)\left(\right.$ red), $C_{3}(\tau)=\sigma_{V}^{2}\left[\exp \left(-\tau^{2} /\left(6 \tau_{s}^{2}\right)\right)-\right.$

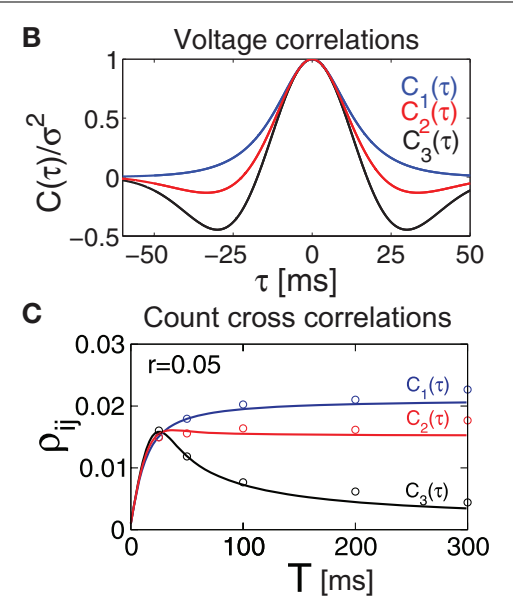

$\left.\tau^{2} /\left(3 \tau_{s}^{2}\right) \exp \left(-\tau^{2} /\left(6 \tau_{s}^{2}\right)\right)\right]\left(\right.$ black). Note, all voltage correlations $C_{i}(\tau)$ share the same correlation time $\tau_{\mathrm{s}}$ but have a different functional form. (C) $\rho_{i j}$ vs. Tfor voltage correlation functions $C_{i}(\tau)$. For all figures the correlation time $\tau_{\mathrm{s}}=10 \mathrm{~ms}$, $v=5 \mathrm{~Hz}, \mathrm{v}=\mathrm{v}_{1}=\mathrm{v}_{2}$; circles denote the corresponding simulation points. $\mathrm{v}_{\text {cond }, i j}(\tau)=\mathrm{v}\left(1+r\left(\tilde{\mathrm{c}}(\tau) k^{2}-\pi \tau_{s}^{2} \tilde{\mathrm{c}}^{\prime \prime}(\tau) / 2\right)\right)$, or

$v_{\text {cond }, i j}(\tau)=v\left(1+r\left(\tilde{\mathrm{c}}(\tau) 2\left|\log \left(2 \pi \nu \tau_{s}\right)\right|-\pi \tau_{s}^{2} \tilde{\mathrm{c}}^{\prime \prime}(\tau) / 2\right)\right)$,

where $\tilde{c}(\tau)=C(\tau) / \sigma_{V}^{2}$ and $k=\psi_{0} / \sigma_{V}$. Equation 24 shows that weak spike correlations are generally firing rate dependent and directly reflect the structure of input correlations $C(\tau)$. Figure $5 \mathbf{A}$ shows three examples of voltage correlations which have the same $\tau_{s}$, but different functional form. All three functional dependencies are reflected in the cross conditional firing rate $v_{\text {cond, } i j}$, but result in markedly different shapes of auto conditional rate $v_{\text {cond }}(\tau)$ (Figures 5A,B). In the next section we study how the functional choice of $C(\tau)$ affects the correlation coefficient.

\section{Count correlations across spike trains}

We now use the spike correlation function obtained above to study the pairwise count covariance.

$$
\begin{aligned}
\operatorname{Cov}\left(n_{\mathrm{i}}(T), n_{\mathrm{j}}(T)\right)= & \int_{-T}^{T} v^{2} r\left(\tilde{c}(t) 2\left|\log \left(2 \pi v_{s}\right)\right|\right. \\
& \left.-\pi \tau_{s}^{2} \tilde{c}^{\prime \prime}(t) / 2\right)(T-|t|) d t
\end{aligned}
$$

which allows to obtain the correlation coefficient for a weakly correlated pair of neurons:

$$
\rho_{i j}=\frac{\operatorname{Cov}\left(n_{i}(T), n_{j}(T)\right)}{\sqrt{\operatorname{Var}\left(n_{i}(T), n_{i}(T)\right) \operatorname{Var}\left(n_{j}(T), n_{j}(T)\right)}}=\frac{\int_{-T}^{T} v r\left(2 \tilde{c}(t)\left|\log \left(2 \pi \nu \tau_{s}\right)\right|-\pi / 2 \tau_{s}^{2} \tilde{c}^{\prime \prime}(t)\right)(T-|t|) / T d t}{\sqrt{\left(1+2 \cdot \int_{0}^{T}\left(v_{\text {cond }}(t)-v_{i}\right)(T-|t|) / T d t\right)\left(1+2 \cdot \int_{0}^{T}\left(v_{\text {cond }}(t)-v_{j}\right)(T-|t|) / T d t\right)}}
$$

functions, correlation coefficient continues increasing slowly for $C_{1}$, remains at the same level for $C_{2}$, but decreases dramatically for $C_{3}$. This latter type of behavior was not observed in previous studies of LIF models (de la Rocha et al. (2007), Suppl.), which focused on the analytically accessible choice of white noise currents and reported a monotonously increasing correlation coefficient in the limit of large $T$. Below we will further consider how dependence of $\rho_{i j}$ on $T$ is influenced by the choice of the form of voltage correlations $C(\tau)$. We will show that some voltage correlation functions can lead to vanishing correlation coefficients in the limit of large bin size $T$.

\section{Vanishing count covariance in the presence of cross correlations}

Count covariances and correlation coefficients rely on the integral of the spike correlation function (Eqs 3 and 7). In cortical neurons, the spike correlation functions can exhibit oscillations and significant undershoots in addition to a correlation peak (Lampl et al., 1999; Galan et al., 2006), this may alter the correlation coefficients and their dependence on bin size $T$. In the weak correlation regime we obtained an analytic expression for $v_{\text {cond, } i j}(\tau)$ (Eqs 24 and 26). This allows us to explore analytically how a change in the functional choice of voltage correlations will influence count correlations. To qualify as a reliable measure of synchrony, count cross correlations between two neurons should reflect primarily
This offers the opportunity to study how changes in the input structure affect spike count correlations. Figure $\mathbf{5}$ shows that correlation coefficient $\rho_{i j}$ depends on both bin size $T$ and the functional form of input correlation function $C(\tau)$. Figure 5C illustrates that different functional form of underlying membrane potential correlations can lead to a strikingly different dependence of $\rho_{i j}$ on the bin size. After an initial increase for all three voltage correlation correlation strength and be independent of the functional form of input correlations. Our framework offers the possibility to test this hypothesis and explore whether previously reported finite correlation coefficients obtained for LIF model using white noise approximation (Shea-Brown et al., 2008) can be generalized to a larger class of input correlations. 
Here we consider spike correlations generated by a voltage correlation function with a substantial undershoot (e.g., as in Figure $1 \mathrm{E}$ in Lampl et al., 1999). For illustration, we could use any voltage correlation function with a large undershoot and vanishing longtimescale variability $\left(\int_{-\infty}^{\infty} C(\tau) d \tau=0\right)$. Besides variance and correlation time, the variability as quantified by $\int_{-\infty}^{\infty} C(\tau) d \tau$ is an important characteristic of every noise process. For analytical tractability we chose the voltage correlation function $\mathrm{C}_{3}(\tau)$ as the normalized second derivative of the function $\tilde{C}_{3}(\tau)=-3 \tau_{s}^{2} \exp \left(-\tau^{2} /\left(6 \tau_{s}^{2}\right)\right)$ :

$C_{3}(\tau)=\sigma_{V}^{2}\left(\exp \left(\frac{-\tau^{2}}{6 \tau_{s}^{2}}\right)-\frac{\tau^{2}}{3 \tau_{s}^{2}} \exp \left(\frac{-\tau^{2}}{6 \tau_{s}^{2}}\right)\right)$

Defined this way, the correlation time of $C_{3}(\tau)$ is $\tau_{s}$ and $\int_{-\infty}^{\infty} C_{3}(\tau) d \tau=0$, which is equivalent to vanishing spectral power for zero frequency. Figure 5 illustrates functional form of $C_{3}(\tau)$ and the corresponding spike cross and auto correlations. The functional form of $C_{3}(\tau)$ fulfills $\lim _{T \rightarrow \infty} \int_{-T}^{T} C_{3}(t)(T-|t|) / T d t=0$. This leads to a vanishing count covariance and spike correlation coefficient for $T \gg \tau_{s}$ (Eq. 26): correlation coefficients computed for small $T$ which are independent of $C(\tau)$ (Eqs 9 and 19), the count correlations computed for $T \geq \tau_{s}$ are a potentially unreliable measure of synchrony.

\section{DISCUSSION}

Unambiguous and concise measures of spike correlations are needed to quantify and decode neuronal activity (Abbott and Dayan, 1999; Greenberg et al., 2008; Krumin and Shoham, 2009). Pairwise spike count correlations are frequently used to describe interneuronal correlations (Averbeck and Lee, 2003; Kass and Ventura, 2006; Greenberg et al., 2008) and many population models are based on these measures (Schneidman et al., 2006; Shlens et al., 2006; Roudi et al., 2009). However, quantitative determinants of count correlations so far remained largely elusive. Here, we used a simple statistical model framework based on the threshold crossings and the flexible choice of temporal input structure to study the signatures of input correlations in count correlations. In general, the details of the spike generating model can have a strong effect on spike correlations, f.e. depending on the dynamical regime, two

$\operatorname{Cov}\left(n_{i}(T), n_{j}(T)\right) / T=\frac{v^{2} r \tau_{s}^{2}\left[12 \mid \log \left(2 \pi \nu \tau_{s}\right)\left(1-\exp \left(\frac{-T^{2}}{6 \tau_{s}^{2}}\right)\right)+\pi\left(1+\exp \left(\frac{-T^{2}}{6 \tau_{s}^{2}}\right)\left(\frac{T^{2}}{3 \tau_{s}^{2}}-1\right)\right)\right]}{T}$

$\Rightarrow \lim _{T / \tau_{s} \rightarrow \infty} \frac{\operatorname{Cov}\left(n_{i}(T), n_{j}(T)\right)}{T} \rightarrow 0, \lim _{T / \tau_{s} \rightarrow \infty} \rho_{i j} \rightarrow 0$

We note that the correlation coefficients and count covariances calculated for this functional form of input correlations can be arbitrarily small if $T \gg \tau_{s}$. This means that the absence of longtimescale variability in the inputs $\left(\int_{-\infty}^{\infty} C_{3}(\tau) d \tau=0\right)$ is equivalent to an absence of long-timescale co-variability in the spike counts. Notably, despite vanishing cross covariance, the variability of the single spike train is maintained and count variance of the single spike train (Eq. 8 ) is finite for $C_{3}(\tau)$ in infinite time bins. Equation 28 implies that experimental correlation coefficients calculated for large time bins are most susceptible to the influence of temporal structure of correlations, and experimental studies focusing on large bin sizes [e.g., $T=192 \mathrm{~ms}$ (Greenberg et al., 2008) or $T=2$ s (Zohary et al., 1994)] could potentially underestimate the correlation strength. For the important regime of low firing rates (Greenberg et al., 2008), where the reset has little influence on the following spike, the threshold model and the LIF model can be expected to yield equivalent results. In this case, Eq. 28 and Figure 5 suggest that finite correlation coefficients, which are increasing with bin size $T$ as reported for the LIF model (de la Rocha et al., 2007) might be limited to the subset of input correlation functions without sizable undershoots. To obtain finite count cross correlations, the voltage correlation functions need to fulfill $\int_{-\infty}^{\infty} C(\tau) d \tau>0$, as $C_{1}(\tau), C_{2}(\tau)$ in Figure 5 do.

Notably, spike count correlations of cortical neurons in vivo can decrease or increase as the length of the time bin increases (Averbeck and Lee, 2003; Smith and Kohn, 2008). These results are consistent with our findings (Figure 5C). Thus, in contrast to the quadratic integrate and fire neurons or two LIF neurons can be more strongly correlated (Vilela and Lindner, 2009). Notably, we found that our statistical framework can replicate many important aspects of neuronal correlations, e.g., nonlinear dependence of spike correlations on the input correlation strength (Binder and Powers, 2001) (Eq. 19), firing rate dependence of weak spike correlations (Svirskis and Hounsgaard, 2003; de la Rocha et al., 2007) (Eq. 20), and independence of spike reliability of the threshold (Mainen and Sejnowski, 1995) (Eq. 21). Furthermore, spike correlations derived here are consistent with many recent results in the commonly used LIF model, e.g., firing rate dependence of weak cross correlations (de la Rocha et al., 2007; Shea-Brown et al., 2008) (Eqs 20 and 24), the influence of noise mean and variance on the firing rates and weak spike correlations (Brunel and Sergi, 1998; de la Rocha et al., 2007; Ostojic et al., 2009) (Eqs 15, 20 and 24), or sublinear dependence of correlation coefficients on input strength (Moreno-Bote and Parga, 2006; de la Rocha et al., 2007) (Eq. 19, Figure 3). While the analytical accessibility of the LIF model is limited by the technically demanding multi dimensional Fokker-Planck equations and provides solutions only in special limiting cases (Brunel and Sergi, 1998; de la Rocha et al., 2007; Shea-Brown et al., 2008), the framework presented here allows for an analytical description of spike correlations.

Measurements of correlation coefficients under different experimental conditions often aim to compare the input correlation strength in pairs of neurons (Greenberg et al., 2008; Mitchell et al., 2009). But is a change in count correlations always indicative of a change in input correlations? The tractability of our framework revealed that spike count correlations can be a poor indicator of input synchrony for some cases of input correlations. Count correlations computed for time bins smaller than 
the intrinsic scale of temporal correlations could be independent of the functional form of input correlations but depend on the firing rate and input correlation strength. This suggests that a change in the correlation coefficient can be related to a change in the input correlation strength, if a firing rate change and a change of intrinsic time scale can be excluded. On the other hand, a change in correlation coefficients computed for large time bins is indicative of a change in input correlation strength only if a change in firing rate, time scale and functional form of input correlations can be excluded. Furthermore, count correlations computed for large time bins can either increase or decrease with increasing time bin or even vanish in a correlated pair. This seemingly contradictory behavior is consistent with the functional dependence of spike count correlations observed in cortical neurons (Averbeck and Lee, 2003; Kass and Ventura, 2006; Smith and Kohn, 2008).

Our results suggest that emulating neuronal spike trains, building efficient population models or determining potential decoding algorithms requires the analysis of full spike correlation functions

\section{REFERENCES}

Abbott, L., and Dayan, P. (1999). The effect of correlated variability on the accuracy of a population code. Neural Comput. 11, 91-101.

Amari, S. (2009). Measure of correlation orthogonal to change in firing rate. Neural Comput. 21, 960-972.

Averbeck, B., and Lee, D. (2003). Neural noise and movement-related codes in the macaque supplementary motor area. J. Neurosci. 23, 7630-7641.

Azouz, R., and Gray, C. M. (1999). Cellular mechanisms contributing to response variability of cortical neurons in vivo. J. Neurosci. 19, 2209-2223.

Binder, M. D., and Powers, R. K. (2001). Relationship between simulated common synaptic input and discharge synchrony in cat spinal motoneurons. J. Neurophysiol. 86, 2266-2275.

Brunel, N., and Sergi, S. (1998). Firing frequency of leaky integrate-and-fire neurons with synaptic current dynamics. J. Theor. Biol. 195, 87-95.

Burak, Y., Lewallen, S., and Sompolinsky, H. (2009). Stimulus-dependent correlations in threshold-crossing spiking neurons. Neural Comput. 21, 2269-2308.

Dayan,P.,andAbbott,L.(2001). Theoretical Neuroscience: Computational and Mathematical Modeling of Neural Systems. Cambridge, MA, The MIT Press.

de la Rocha, J., Doiron, B., Shea-Brown, E., Josic, K., and Reyes, A. (2007). Correlation between neural spike trains increases with firing rate. Nature 448, 802-806.

Destexhe, A., Rudolph, M., and Pare, D. (2003). The high-conductance state of neocortical neurons in vivo. Nat. Rev. Neurosci. 4, 739-751.
Fourcaud, N., and Brunel, N. (2002). Dynamics of the firing probability of noisy integrate-and-fire neurons. Neural Comput. 14, 2057-2110.

Galan, R., Fourcaud-Trocme, N., Ermentrout, G., and Urban, N. (2006). Correlation-induced synchronization of oscillations in olfactory bulb neurons. J. Neurosci. 26, 3646-3655.

Greenberg, D., Houweling,A., and Kerr, J. (2008). Population imaging of ongoing neuronal activity in the visual cortex of awake rats. Nat. Neurosci. 11, 749-751.

Jung, P. (1995). Stochastic resonance and optimal design of threshold detectors. Phys. Lett. A 207, 93-104.

Kass, R., and Ventura, V. (2006). Spike count correlation increases with length of time interval in the presence of trial-to-trial variation. Neural Comput. 18, 2583-2591.

Kriener, B., Helias, M., Aertsen, A., and Rotter, S. (2009). Correlations in spiking neuronal networks with distance dependent connections. J. Comput. Neurosci. 27, 177-200.

Krumin, M., and Shoham, S. (2009). Generation of spike trains with controlled auto- and cross-correlation functions. Neural Comput. 21, 1642-1664.

Lampl, I., Reichova, I., and Ferster, D. (1999). Synchronous membrane potential fluctuations in neurons of the cat visual cortex. Neuron 22, 361-374.

Leadbetter, M., Lindgren, G., and Rootzen, H. (1983). Extremes and Related Properties of Random Sequences and Processes. Springer, New York (Springer Series in Statistics Edition).

in order to compute unambiguous spike count correlations. In particular, spike count coefficients computed for time bins larger than intrinsic timescale of correlations can be an ambiguous estimate of input cross correlations in a neuronal population with potentially heterogeneous distribution of input structures. Furthermore, the details of the spike generation model can be very influential for the transfer of current correlations to spike correlations, and the analytical results obtained here could facilitate quantitative comparisons between different types of models and between models and real neurons, by providing a maximally tractable limiting case for future studies.

\section{ACKNOWLEDGMENTS}

We wish to thank M. Gutnick, I. Fleidervich, S. Ostojic and A. Malyshev for fruitful discussions and the Bundesministerium für Bildung und Forschung (\#01GQ0430,\#01GQ07113), Goettingen Graduate School for Neurosciences and Molecular Biosciences, German-Israeli Foundation (\#I-906-17.1/2006), University of Connecticut and the Max Planck Society for financial support.

Macke, J., Berens, P., Ecker, A., Tolias, A., and Bethge, M. (2009). Generating spike trains with specified correlation coefficients. Neural Comput. 2 , 397-423.

Mainen, Z. F., and Sejnowski, T. J. (1995). Reliability of spike timing in neocortical neurons. Science 268, 1503-1506.

Mitchell, J. F., Sundberg, K. A., and Reynolds, J. H. (2009). Spatial attention decorrelates intrinsic activity fluctuations in macaque area $\mathrm{v} 4$. Neuron $63,879-888$.

Moreno-Bote, R., and Parga, N. (2006) Auto- and crosscorrelograms for the spike response of leaky integrate-andfire neurons with slow synapses. Phys. Rev. Lett. 96, 028101.

Ostojic, S., Brunel, N., and Hakim, V. (2009). How connectivity, background activity, and synaptic properties shape the cross-correlation between spike trains. J. Neurosci. 29, 10234-10253.

Perkel, D. H., Gerstein, G. L., and Moore, G. P. (1967). Neuronal spike trains and stochastic point processes. ii. simultaneous spike trains. Biophys. $J$. 7, 419-440.

Prichard, D., and Theiler, J. (1994). Generating surrogate data for timeseries with several simultaneously measured variables. Phys. Rev. Lett. 73, 951-954.

Rice, S. O. (1954). Mathematical analysis of random noise. In Selected Papers on Noise and Stochastic Processes, N. Wax, ed. (New York, Dover).

Roudi, Y., Nirenberg, S., and Latham, P. (2009). Pairwise maximum entropy models for studying large biological systems: when they can work and when they can't. PLoS Comput. Biol.,
5, e1000380. doi:10.1371/journal. pcbi. 1000380 .

Schneidman, E., Berry, M. J., Segev, R. and Bialek, W. (2006). Weak pairwise correlations imply strongly correlated network states in a neural population. Nature 440, 1007-1012.

Shea-Brown, E., Josic, K., de la Rocha, J., and Doiron, B. (2008). Correlation and synchronytransferinintegrateand-fire neurons: basic properties and consequences for coding. Phys. Rev. Lett. 100, 108102.1-108102.4.

Shlens, J., Field, G., Gauthier, J., Grivich, M., Petrusca, D., Sher, A., Litke, A., and Chichilnisky,E. (2006). The structure of multi-neuron firing patterns in primate retina. J. Neurosci. 26, 8254-8266.

Smith, M.A., and Kohn,A. (2008). Spatial and temporal scales of neuronal correlation in primary visual cortex. $J$. Neurosci. 28, 12591-12603.

Steinmetz, P. N., Roy, A., Fitzgerald, P. J., Hsiao, S. S., Johnson, K. O., and Niebur, E. (2000). Attention modulates synchronized neuronal firing in primate somatosensory cortex. Nature 404, 187-190.

Stopfer, M., Bhagavan, S., Smith, B. H., and Laurent, G. (1997). Impaired odour discrimination on desynchronization of odour-encoding neural assemblies. Nature 390, 70-74.

Stratonovich, R. (1964). Topics in the Theory of Random Noise, Vols I-II. New York, Gordon and Breach.

Svirskis, G., and Hounsgaard, J. (2003). Influence of membrane properties on spike synchronization in neurons: theory and experiments. Network 14, 747-763.

Takahashi, S., and Sakurai, Y. (2006). Dynamic synchrony of firing in the 
monkey prefrontal cortex during working-memory tasks. J. Neurosci. 26, 10141-10153.

Tchumatchenko, T., Malyshev, A., Geisel, T., Volgushev, M., and Wolf, F. (2010). Correlations and synchrony in threshold neuron models. Phys. Rev. Lett. 104, 058102.

Tetzlaff, T., Rotter, S., Stark, E., Abeles, M., Aertsen, A., and Diesmann, M. (2008). Dependence of neuronal correlations on filter characteristics and marginal spike train statistics. Neural Comput. 20, 21332184.
Vaadia, E., Haalman, I., Abeles, M., Bergman, H., Prut, Y., Slovin, H., and Aertsen, A. (1995). Dynamics of neuronal interactions in monkey cortex in relation to behavioural events. Nature 373, 515-518.

Vilela, R. D., and Lindner, B. (2009). Comparative study of different integrate-and-fire neurons: spontaneous activity, dynamical response, and stimulus-induced correlation. Phys. Rev. E 80, 031909.

Wolfram Research (2009). As Implemented in MATHEMATICA 5.2. Wolfram Research.
Zohary, E., Shadlen, M. N., and Newsome, W.T. (1994). Correlated neuronal discharge rate and its implications for psychophysical performance. Nature 370, 140-143.

Conflict of Interest Statement: The authors declare that the research was conducted in the absence of any commercial or financial relationships that could be construed as a potential conflict of interest.

Received: 15 November 2009; paper pending published:07 December 2009; accepted:
05 February 2010; published online: 08 April 2010.

Citation: Tchumatchenko T, Geisel T, Volgushev Mand WolfF (2010) Signatures of synchrony in pairwise count correlations. Front. Comput. Neurosci. 4:1. doi: 10.3389/neuro.10.001.2010

Copyright (C) 2010 Tchumatchenko, Geisel, Volgushev and Wolf. This is an open-access article subject to an exclusive license agreement between the authors and the Frontiers Research Foundation, which permits unrestricted use, distribution, and reproduction in any medium, provided the original authors and source are credited. 